\title{
A very rare case of adult-type granulosa cell tumor
}

\author{
Roberto Giulianelli $^{1}$, Gabriella Mirabile ${ }^{1}$, Giorgio Vincenti ${ }^{1}$, Francesco Pellegrino ${ }^{2}$, Giuseppe Soda ${ }^{2}$ \\ ${ }^{1}$ Roman Urological Association, Division of Urology, Nuova Villa Claudia, Rome, Italy; \\ ${ }^{2}$ Dipartimento di Medicina Sperimentale Policlinico Umberto I, Università "Sapienza" di Roma, Rome, Italy.
}

\begin{abstract}
Summary Granulosa cell tumor (GST) of the testis is a rare neoplasm. Here we describe a case of an adult type GST. More than a year after surgical treatment, without any other treatment, the patient is alive without sign of disease.
\end{abstract}

KEY WORDS: Granulosa cells; Tumor; Testis; Adult-type.

Submitted 19 October 2014; Accepted 31 December 2014

\section{INTRODUCTION}

Sex cord-stromal tumors (SCSTs) comprise 4-5\% of all testicular tumors. This group of tumors includes Leydig cell, Sertoli and Granulosa cell tumors, in addition to mixed cell types and undifferentiated tumors, with the Leydig cell group as the most frequent subtype ( $75 \%$ of all SCSTs)

Granulosa cell tumors (GSTs) are even more rare, and they have been divided in two distinct groups: the juvenile type and the adult type. The adult-type is an extremely rare entity, with, to the best of our knowledge, only 31 cases reported in literature. Here we describe a case of an 80 years old Caucasian male with an adulttype GST located in the right testis. This is the oldest man with a diagnosis of GST of the testis reported.

\section{Materials AND METHOdS}

An 80 year old white male presented with an inguinal swelling, during prostate cancer follow up, after a radical prostatectomy performed 14 years before. He also presented with a light painful gynecomastia, but he was taking bicalutamide. The physical examination was otherwise normal.

Palpation and ultrasound examination revealed a tumor of the right testis in the inguinal canal. The testis had an irregular morphology, with polilobulated hedges and disomogeneous ultrasound architecture. There were also multiple cysts, the biggest one located in the posterior wall $(36 \times 48 \times 45 \mathrm{~mm})$. The left testis was smaller than normal $(7 \mathrm{cc})$.

His preoperative blood tests (including tumor markers such as LDH, beta-HGC and AFP) were normal. The abdominal and thoracic CT scans did not show any significant lymph node enlargements or distant metastases.
Surgical excision was decided and right inguinal orchiectomy was performed. The surgical specimen consisted of the right testis measuring $6 \times 6 \times 5 \mathrm{~cm}$, in continuity with the spermatic cord measuring $\mathrm{cm}^{2}$ in length.

On cross-sections the testis was totally replaced by a tumor partly solid, partly cystic, measuring $6 \mathrm{~cm}$ in diameter, with homogeneous appearance and yellowish colour. No necrosis or hemorrhage were seen.

Histologically, the tumor was composed of medium-size atypical cells with a diffuse growth pattern. The nuclei were round to oval, with evident nucleoli, sometimes with nuclear grooves, and scanty cytoplasm (Figure 1).

No other recognizable sex cord tumor component was identified. Mitoses were approximately 4 x 10 HPF.

There were no hemorrhage, necrosis or vascular invasion; the tunica albuginea, the spermatic cord and the margins of surgical resection were free of tumor.

The remaining testicular parenchyma was atrophic. The immunoprofile of the neoplastic cells was vimentin positive, inhibin positive, cytokeratin negative, LCA negative, CD99 negative, PLAP negative and Melan-A negative. Histochemical reticulum staining was also positive. The microscopic and immunohistochemical findings were consistent with the diagnosis of adult-type granu-

\section{Figure 1.}

The tumor shows a diffuse growth pattern; the cells are characterized by round/oval nuclei, evident nucleoli, nuclear grooves and occasional mitoses.

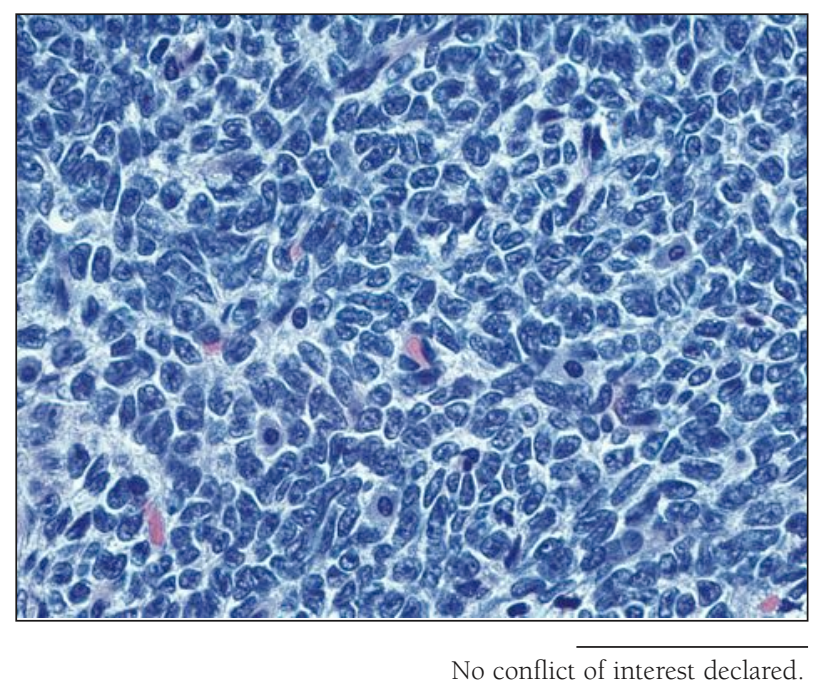


losa cell tumor. Post-operative course was free of complications and after one-year of follow-up a total body CT scan confirmed that the patient is still disease-free.

\section{Discussion}

SCSTs are rare neoplasms of the testis (1) and GCTs are even more rare, especially if we consider the adult-type. Generally more than half of the patients with adult type GSTs presented with slow and painless testicular enlargement, with a variable period of growth (2) and with a range in age at presentation between 16 and 77 years (2). Only in a small proportion of cases gynecomastia was reported. A specific diagnosis of adult-type granulosa cell tumor require that all or almost all the neoplasm have granulosa cell features (medium size, ovoid-elongated muclei, nuclear grooves).

This because the presence of tubular differentiation indicates that the tumor is of Sertoli cell type, or mixed/unclassified sex-chord stromal tumor. Obviously many differential diagnosis have to be considered. Due to the rarity of these tumors, the optimal management of these patients is still difficult to establish, as does the clinica behaviour, which may be potentially malignant. Kim et al. established six histopathological criteria for malignancy and metastatic potential for Leydig cell tumors: tumor size $>5 \mathrm{~cm}$, necrosis, angiolymphatic invasion, infiltrating margins, moderate/severe nuclear atypia, and $>5$ mitoses per $10 \mathrm{HPF}$. Afterwards all these criteria have been accepted for other SCSTs, and so they are valid for GCTs as well. In our case, according to these criteria, the only histopathological feature predictive of metastatic potential was the major diameter of the lesion $(6 \mathrm{~cm})$. Adult type GCTs can follow an aggressive course; most frequently, metastases are found in the retroperitoneal lymph nodes, but also other sites have been described as liver, bones and lungs (1). However at present there is no consensus regarding discriminating criteria in order to predict a malignant behaviour of these tumors. Silberstein et al. evaluated the management options of SCSTs of the testis, referring to the high-risk features mentioned above (3). They noticed that in their cohort of patients with $\leq 1$ high risk factor, who received radical orchiectomy without adjuvant therapy, none of them developed disease recurrence or progression. Other investigators, in a similar cohort of patients, most of them having zero risk factors, observed no cases of metastases after radical orchiectomy alone. All these data suggest that following orchiectomy, surveillance alone, is the preferred management for patients with $\leq 1$ high risk factor, considering the very low likelihood of disease progression/recurrence (3) as in the case we described. Given that our patient presented with only one high risk factor (i.e. a major diameter of tumor $>5 \mathrm{~cm}$ ) and given and considering the reported data in the literature (3), in our opinion the most reasonable management following radical orchiectomy was a strict and long term surveillance alone. However initial treatment for these tumors is radical orchiectomy, with the option of a retroperitoneal lymphadenectomy in cases where metastases are suspected.

\section{Conclusions}

In conclusion we reported a very rare case of adult type granulosa cell tumor of the testis, an entity that brings many problems in terms of management and outcome predictions.

We recommend strict and long-term follow-up as recurrence and metastases have been reported years after diagnosis.

\section{REFERENCES}

1. Colecchia M, Mikuz G, Algaba F. Rare tumors of the testis and mesothelial proliferation in the tunica vaginalis. Tumori. 2012; 98:270-273.

2. Ditonno P, Lucarelli G, Battaglia M, et al. Testicular granulosa cell tumor of the adult type: a new case and review of the literature. Urologic Oncol. 2007; 25:322-325.

3. Silberstein JL, Bazzi WM, Vertosick E, et al. Clinical outcomes of local and metastatic testicular sex cord-stromal tumors. J Urol. 2014; 192:415-9.

\author{
Correspondence \\ Roberto Giulianelli, MD (Corresponding Author) \\ roberto.giulianelli@virgilio.it \\ Gabriella Mirabile, MD \\ Giorgio Vincenti, $M D$ \\ Roman Urological Association \\ Division of Urology, Nuova Villa Claudia \\ Via Flaminia Nuova 280 - 00100 Rome, Italy \\ Francesco Pellegrino, MD \\ Giuseppe Soda, MD \\ Dipartimento di Medicina Sperimentale Policlinico Umberto I, \\ Università "Sapienza" di Roma \\ Viale Regina Elena 324 - 00161 Rome, Italy
}

